\title{
ISPAD Annual Conference 2019 Highlights
}

\author{
Ananta Addala $^{1}$ | Christine March ${ }^{2}$ | Brynn Marks ${ }^{3}$ | Kalie Tommerdahl ${ }^{4}$ | \\ Jenna Shapiro ${ }^{5}$ | Elizabeth Oyenusi ${ }^{6}$ | Lauren McClure Yauch ${ }^{7}$ | \\ Eveline R. Goethals ${ }^{8}$ | Peerzada Ovais Ahmad ${ }^{9}$ | Sara Adhami ${ }^{10}$ | May $\mathrm{Ng}^{11}$ | \\ Sarah Ehtisham ${ }^{9}$ | Juliana Chizo Agwu ${ }^{12}$ \\ ${ }^{1}$ Division of Pediatric Endocrinology, Stanford University, Stanford, California \\ ${ }^{2}$ Division of Pediatric Endocrinology, UPMC Children's Hospital of Pittsburgh, Pittsburgh, Pennsylvania \\ ${ }^{3}$ Division of Endocrinology, Children's National Hospital, Washington, District of Columbia \\ ${ }^{4}$ Division of Pediatric Endocrinology, Children's Hospital of Colorado | Barbara Davis Center, Aurora, Colorado \\ ${ }^{5}$ Pritzker Department of Psychiatry and Behavioral Medicine, Ann \& Robert H. Lurie Children's Hospital of Chicago, Chicago, Illinois \\ ${ }^{6}$ Endocrinology and Metabolism Unit, Department of Paediatrics, College of Medicine, University of Lagos/Lagos University Teaching Hospital Idi-Araba, Lagos, \\ Nigeria \\ ${ }^{7}$ Division of Pediatric Endocrinology, University of Minnesota, Minneapolis, Minnesota \\ ${ }^{8}$ Pediatric Research, Joslin Diabetes Center, Harvard Medical School, one Joslin PI, Boston, Massachusetts \\ ${ }^{9}$ Department of Endocrinology, Sher-i-Kashmir Institute of Medical Sciences (SKIMS), Srinagar, India \\ ${ }^{10}$ Endocrinology Department, Mediclinic City Hospital, Dubai, United Arab Emirates \\ ${ }^{11}$ Department of Paediatrics, Southport and Ormskirk Hospitals NHS Trust, Honorary Senior Lecturer, University of Liverpool, Liverpool, UK \\ ${ }^{12}$ Department of Pediatrics, Sandwell and West Birmingham NHS Trust, Institute of Clinical Sciences, College of Medicine and Dental Sciences, University of \\ Birmingham, Birmingham, UK

\section{Correspondence} \\ Juliana Chizo Agwu, Department of Paediatrics, Sandwell and West Birmingham NHS Trust, West Bromwich B71 4HJ, UK. \\ Email: chizo.agwu@nhs.net
}

\section{1 | PRESIDENT'S SPEECH}

The incidence of type 1 diabetes (T1DM) and type 2 diabetes (T2DM) rate has increased worldwide, affecting more than 1.1 million children. In T1DM, a dramatical increase in hemoglobin A1c (HbA1c) occurs specifically in late adolescence and young adulthood, putting youth at risk for short- and long-term complications. Over 90000 children with diabetes live in poverty, without food or insulin security, and with high rates of diabetes-related complications. Therefore, social and economic factors continued improvements in technology, education of families, adequate access to insulin, and food security are important components in the management of pediatric T1DM. Although technological advances, such as continuous glucose monitoring (CGM), insulin pump, and hybrid closed-loop systems help in optimizing glycemic control, access to these vary by country, income, and by race/ethnicity. Hence, there is a call for action with regard to these continued challenges in achieving more optimal glycemic control across the globe.

\section{2 | PLENARY SESSION-BETA CELLS: PAST, PRESENT, AND FUTURE}

Historically, T1DM prevention trials have focused on immune modulation as a means of prevention or slowing the progression of T1DM. The network for Pancreatic Organ Donors with Diabetes study which aims to examine the underlying pathophysiology of T1DM by examining pancreatic tissue has shown that the degree of insulitis is milder and more heterogeneous in humans compared with murine models; insulitis seems to be more pronounced in certain islets rather than others. The islet cells are abnormal in individuals with T1DM due to both diminishing blood supply and increased beta cell stress. Matched for age and body mass index (BMI), the pancreas of individuals with T1DM is smaller at diagnosis compared to that of healthy controls. These insights into the pathology of the pancreas, islets, and beta cells provoke interesting questions as to the true etiology for this disease; does this disease result from an autoimmune attack of healthy beta cells or an immune-mediated attack of inherently damaged beta cells? 
Ultimately, a combination of factors is likely contributing to disease, and future therapeutic targets should address beta cell function in addition to immune response. One such therapeutic approach may be with the use of embryonic stem cells to create "designer islets." Embryonic stem cells can be induced to form beta-cell predominant islets which are quite stable and respond to a glucose challenge in animal models with appropriate insulin secretion. Specially encapsulated embryonic stem cells are currently under investigation to offer both a therapeutic and mechanistic insight in the pathophysiology of T1DM.

\section{3 | SYMPOSIUM-IMPLEMENTING TECHNOLOGIES AND OVERCOMING BARRIERS TO USE}

Two hybrid-closed insulin delivery systems are currently available: the MiniMed 670G insulin pump with Guardian 3 sensor (which when in auto mode, automatically adjusts basal insulin delivery every 5 minutes based on sensor glucose levels) and the Tandem's ControlIQ automated insulin delivery (AID) system which combines the t:slim X2 insulin pump, the Dexcom G6 CGM, and an algorithm that adjusts basal insulin delivery and gives automatic correction boluses. The CARES (calculate, adjust, revert, educate, sensor/share) paradigm can be utilized to help guide clinicians in making changes to the management regimen. Calculate refers to how the system calculate the insulin doses it aims to deliver (ie, total daily dose for MiniMed 670G vs basal rates for control IQ). Adjust indicates the parameters that the clinician can modify for improved control (ie, insulin to carbohydrate ratio and insulin action time for MiniMed 670G vs basal rates, insulin to carbohydrate ratio, and insulin sensitivity factor for control IQ). Revert refers to conditions that will cause the system to revert to non-AID (ie, prolonged dysglycemia and loss of sensor data for MiniMed $670 \mathrm{G}$ vs loss of sensor data for control IQ). Educate refers to the need to educate patients and providers on how to be successful with the idiosyncrasies of each system (ie, follow all prompts to stay in auto mode and modify carbohydrate coverage if the system needs to be more aggressive for MiniMed 670G vs following bolus recommendations, setting a sleep schedule, and reading prompts carefully for control IQ). Sensor/Share requires understanding of the requirements for successful sensor use and if remote monitoring is available (ie, calibrations are needed and there are no remote monitoring for MiniMed 670G vs no calibrations needed and remote monitoring available for control IQ).

\section{4 | SYMPOSIUM-CURRENT THINKING ABOUT MICROVASCULAR COMPLICATIONS}

Diabetic kidney disease (DKD) is rising in prevalence and disproportionately impacts young women with T2D. In a normal kidney there is a balance between energy demand and expenditure, but in kidneys that are impacted by diabetes, glucosuria and exposure to endogenous or exogenous hyperinsulinism secondary to treatment causes increased renal energy expenditure through increased $\mathrm{Na}+/ \mathrm{K}+$
ATPase expression and activity, increased sodium glucose linked transporter 2 (SGLT2) expression, and increased glucose and $\mathrm{Na}$ + uptake at SGLT2. This results in hyperfiltration, an acute increase in glomerular filtration rate. Diabetes is also associated with increased insulin resistance and mitochondrial dysfunction and this shifts renal fuel utilization toward free fatty acid oxidation which has a low yield of adenosine triphosphate (ATP) per the amount of oxygen $\left(\mathrm{O}_{2}\right)$ consumed. The combination of a decreased amount of ATP generated and an increased amount of ATP consumed results in an ATP deficit which produces increased renal $\mathrm{O}_{2}$ consumption and subsequent hypoxia and this is hypothesized to drive the development of DKD. However, human data linking early DKD with increased renal oxygen consumption and renal hypoxia is missing though there are studies underway. Studies have suggested an up to 22-fold decreased risk of DKD at 5 years with bariatric surgery compared to medical management. Further understanding of the molecular and metabolic mechanisms of renoprotection that are impacted by bariatric surgery could help us uncover novel targets for the development of novel nonsurgical therapies.

Guidelines for retinopathy screening in T1DM recommend a comprehensive, dilated fundus examination after the age of 10 years or within 3 to 5 years of a diabetes diagnosis, followed by annual examinations whereas for T2DM, screening is recommended immediately after diagnosis and then annually. Effective treatments for diabetic retinopathy include Anti-vascular endothelial growth factor (anti-VEGF) agents, laser therapy, and steroids.

Diabetic autonomic neuropathy (AN) manifests in the gastrointestinal, urogenital, sudomotor, cardiac, and neuroendocrine systems. Management of AN primarily focuses on optimization of hemoglobin $\mathrm{A} 1 \mathrm{c}(\mathrm{HbA1c})$, lifestyle interventions to maintain a healthy BMI.

\section{5 | SYMPOSIUM-SODIUM GLUCOSE LINKED TRANSPORTER 2 (SGLT2) INHIBITORS-DO THEY HAVE A ROLE IN PEDIATRIC DIABETES CARE?}

The use of SGLT2 inhibitors is one of the novel approaches being studied as adjunct to insulin therapy in T1DM and T2DM. SGLT2 receptors are expressed in the proximal collecting tubule of the kidney and these agents have been shown to offer long-term cardiovascular and renal protection by decreasing hyperfiltration in the remaining nephrons and normalizing the glomerular pressure. SGLT2 numbers and activity are up-regulated even with short term exposure to hyperglycemia. SGLT2 inhibitors have demonstrated effective glucoselowering benefits and reductions in CV mortality and hospitalization for heart failure in adults with T2DM. Studies demonstrate an improvement in $\mathrm{HbA} 1 \mathrm{c}$, decreased daily insulin requirements, improved time in range (TIR), decrease of 3 to $4 \%$ in body weight and reductions in hypoglycemia. Side effects include a two to fourfold increase in diabetic ketoacidosis (DKA), genital infection (vulvovaginitis /balanitis), and urinary tract infections as well as some emerging concerns about the impact on bone health with prolonged use in a 
pediatric population. It is hypothesized that the cause of the emergent DKA with SGLT2 inhibitor agents stems from the need to lower insulin dose to avoid further hypoglycemia in view of the glucose lowering effects of these agents. Caution needs to be exercised in the use of SGLT2 inhibitors in pediatric patients with DM. Real world studies are needed to help weigh the risk and benefit of incorporating SGLT2 inhibitors in to the management of pediatric T1DM and T2DM patients.

\section{6 | SYMPOSIUM-GOING BEYOND ROUTINE CARE}

The goals of food management in T1DM are to achieve optimal glycemic control. Meals that are high in fat and protein have been shown to increase postprandial glucose levels for many hours after consumption. Incorporating protein with carbohydrate in a meal can prevent hypoglycemia during and after exercise. Various insulin dosing methods for fats and proteins have been described such as the Pankowska Formula where additional insulin is calculated using a fat/protein unit (1 unit of insulin is given for every $100 \mathrm{kcal}$ in the meal from fat and protein). Another method described is the Food Insulin Index which is a measure of postprandial insulin responses to a reference food. Although methods for carbohydrate counting have been validated, at present, insulin dosing for fats and proteins are not standardized.

Diabetes apps that make life a little easier will help reduce the mental labor and burden of the condition. CGM apps have now replaced receivers. They offer improved data analysis and reduce the burden of having to download data regularly. Apps can also send automated weekly emails and notifications of data insights and provide individualized remote coaching.

\section{7 | SYMPOSIUM-IT'S NOT JUST ABOUT} THE A1C

$\mathrm{HbA1c}$ has long been the gold standard for assessing glycemic control. Many advantages can be described: it can be measured with a high degree of precision, it is a valuable measure of hyperglycemia for reporting group and population averages, and has strong association with chronic vascular complications. However, important drawbacks include the inter-individual variation in $\mathrm{HbA1c}$ mean glucose relationship and that it cannot assess patterns of glycemia-the same $\mathrm{HbA1c}$ can be associated with very different patterns of glycemia and it takes 2 to 3 months to get a measure of glycemia. Conditions such as hemoglobinopathy, iron deficiency anemia affect the HbA1c measurement. Data from the T1DM exchange demonstrated a $0.5 \%$ difference in mean $\mathrm{HbA} 1 \mathrm{c}$ between African Americans and Caucasians accounted for by race alone. Therefore, management approaches based on HbA1c alone could be overly aggressive for some individuals with diabetes, and not aggressive enough for others.
CGM metrics for hyperglycemia, hypoglycemia, TIR, mean glucose, and glycemic variability have been defined. TIR is defined as the time between 70 and $180 \mathrm{mg} / \mathrm{dL}$ and ideal target is a TIR of more than $70 \%$. TIR is strongly correlated with vascular complication rates, similar to $\mathrm{HbA1c}$ and has been validated as an outcome measure for clinical trials. Ambulatory glucose profile shows the patterns of glucose levels that can be used to make targeted changes in diabetes management. The new term for estimated HbA1c from CGM is glucose management indicator.

\section{8 | SYMPOSIUM: HOW AND WHY EXERCISE MATTERS}

Engaging in regular exercise is beneficial for youth with T1DM, but dysglycemia associated with being physically active can make glycemic control during and after exercise challenging. Whereas aerobic exercise tends to lower glucose levels, anaerobic exercise can cause glucose levels to rise. Providers often recommend activities that contain a mix of aerobic and anaerobic exercise to prevent dysglycemia. Given that certain types of exercise can increase blood glucose, any decision-making about carbohydrate intake before and during exercise needs to be informed by the type and intensity of exercise. Wearables, remote monitoring, and apps that incorporate information related to heart rate, insulin delivery, and activity level can provide data to refine clinical guidelines.

Mice studies show that maternal and paternal exercise improved glucose tolerance in offspring of both genders, and both parents exercising was associated with even greater improvements. Differences in hepatic gene expression observed in offspring embryos explain the link between maternal exercise and offspring metabolic control. Findings could have implications for the prevention of obesity in offspring.

\section{9 | SYMPOSIUM-CARDIOVASCULAR DISEASE (CVD) RISK-AN OFTEN- OVERLOOKED PEDIATRIC CONCERN}

Individuals with T1DM diagnosed before age 10 years have a reduction in life expectancy of 17.7 years for women and 14.2 years for men, largely due to CVD. While the DCCT study demonstrated the long-term cardiovascular benefit of improved glycemia in T1DM, the ACCORD study has shown that there is an increased risk of weight gain with improved glycemic control and this also increases the risk for development of insulin resistance and CVD. With elevated BMI in T1DM, derangements in leptin, highly sensitive C-reactive protein, heart rate, blood pressure, brachial artery distensibility, the athlete's maximum capacity to utilize oxygen in a graded exercise test $\left(\mathrm{vO}_{2}\right.$ peak), and pulse wave velocity approximate that of T2DM. Individuals with T2DM have higher levels of triglycerides, high-density lipoproteins, adiponectin, alanine aminotransferase, microalbuminuria, and glomerular filtration rate than obese individuals with T1DM. 
The Adolescent T1DM Cardio-Renal Intervention Trial (AdDIT) study set out to investigate whether the upper tertile of albumin:creatinine ratio (ACR) predicts cardiovascular complications and whether it was modifiable. A total of 4407 adolescents with T1DM were divided into tertiles of ACR. At baseline, the high ACR group had hyperfiltration as evidenced by lower cystatin $C$, which is inversely related to GFR. Hyperfiltration may be a risk factor for cardiovascular disease. They also had higher BP and cholesterol levels. Disappointingly the ACR was not significantly affected by ACE inhibitor or statin therapy, or the combination of the two.

\section{0 | SYMPOSIUM-OBESITY AND TYPE 2 DIABETES}

The TODAY (Treatment Options for Diabetes in Adolescents and Youth) and RISE (Restoring Insulin SEcretion) studies have described the aggressive natural history of T2DM in youth, despite pharmacotherapy and lifestyle modifications. Results from the TEENLabs study, a multicenter study of 242 adolescents who underwent bariatric surgery, demonstrated near complete remission of T2DM and associated comorbidities, which persisted at 3 years following surgery. Indications for bariatric surgery include elevated $\mathrm{BMI} \geq 40 \mathrm{~kg} / \mathrm{m}^{2}$ or $140 \%$ of the 95th weight percentile for age with coexisting significant obesity complications, such as T2DM. Sleeve gastrectomy (more preferred in recent years) and Roux-en- $Y$ (gastric bypass) are the common surgical options. Complications of gastric bypass include micronutrient deficiencies. Contraindication to bariatric surgery include medically treatable cause of obesity, ongoing substance abuse, conditions that will interfere with post-operative recovery, or planned pregnancy within 12 to 18 months of procedure.

\section{1 | SYMPOSIUM: THE BRAIN AND DIABETES}

The focus of neuroimaging in the study of T1DM is to better understand the neurobiological basis of altered cognition in response to glycemic extremes. Some studies have shown that glycemic extremes are associated with greater decrease in brain volume over a 2- to 3-year period. Both hyperglycemia and hypoglycemia were associated with brain changes. Events at diagnosis have impact on brain development years later, specifically hyperglycemia and DKA. Future studies are needed to evaluate the impact of these changes on cognitive function.

\section{2 | PLENARY SESSION: NEW THINKING ON GENETICS, INSULIN RESISTANCE AND PEDIATRIC DIABETES}

T1DM patients are more likely to have common genetic variants with small effects; those with negative autoantibodies may be more fruitful to study as they may have missed monogenic diabetes. There are some genetic causes of antibody positive diabetes, for example, FOXP3 mutations but these are rare. In the Norwegian Childhood diabetes registry, all antibody negative cases were screened by exome sequencing and in $6.5 \%$ variants in monogenic diabetes genes were identified; mostly in the HNF1a gene but also rarer MODY variants. One of the clear advantages of a genetic diagnosis is the potential for alteration of treatment, for example, those with KCNJ11 mutations are amenable to sulfonylurea treatment and can come off insulin with improvement in $\mathrm{HbA} 1 \mathrm{c}$. The genetics of T2DM is a large field with more than 500 loci that have been identified with common and intermediate effect low frequency variants conferring some risk.

\section{3 | SYMPOSIUM-THE ISLETS, THE MICROBIOME, AND MORE}

A combination of genetic, environmental, and immunologic factors contributes to the loss of beta cell mass and ultimately progression to T1DM. Most clinical trials have targeted the immune process to reverse or slow T1DM with a minimal or transient effect in most cases. Emerging therapies for clinical trials may begin to target the beta cell, the microbiome, or viruses instead. Using mouse models, there is evidence that increasing beta cell proliferation prior to the time of T-cell education can lead to a protective effect due to a modulated immune response and reduced priming of T-cells. In both human and mouse studies, altered gut microbiota are associated with T1DM and may play a role in immune activation through the production of altered metabolites, specifically short chain fatty acids. The Diabetes Virus Detection Study (DiViD) study which examined fresh pancreatic tissue at the diagnosis of type 1 diabetes (T1D) for the presence of viruses has provided suggestive evidence for the role of viruses, through both specific immune response patterns in the islets and the presence of enteroviral genetic material in cells. All of these discoveries add to the increasingly complex understanding of the pathophysiology of this disease, allowing for new opportunities to identify novel therapeutic targets.

\section{4 | SYMPOSIUM-T1DM CARE IN DEVELOPED AND DEVELOPING NATIONS}

T1DM is challenging to manage even in well-resourced settings. Data from the United States T1DM Exchange registry show HbA1c in the cohort has worsened in the last decade for all age groups despite a drastic increase in CGM use in young children. The Swedish national registry on the other hand report continued improvement in overall glycemic control of their cohort. One of the reasons for this improvement is a system where all pediatric diabetes centers are benchmarked against each other and there is an ongoing process of quality improvement which involves all team members and patients.

In less resourced countries, self-monitoring of blood glucose (SMBG) with even two tests/day is generally more expensive than 
insulin. Mortality is high among young people with new onset T1DM. Survivors usually have high HBA1c, poor quality of life and develop early complications. "Life for a Child" has proposed a concept of "intermediate care"; a care level in-between the "Comprehensive, Guidelines-based Care" that is practiced in well-resourced nations, and "Minimal care" (twice daily insulin injections, clinic visit glucose monitoring, $\mathrm{HbA} 1 \mathrm{c}$ of 12 to $14 \%$, and little to no screening for T1DMrelated complications). The proposed intermediate care model is intended for developing nations only with the aim to incorporate multiple daily injections, home glucose monitoring, complications screening, and target $\mathrm{HbA} 1 \mathrm{c}$ that is 8.0 to $9.5 \%$ as a starting point. Advocacy is required to provide this level of care and establish sustainability.

\section{5 | SYMPOSIUM-TECHNOLOGIES AND DIABETES - WHERE ARE WE TODAY?}

Studies indicate that frequent clinic visits and insulin titration are essential for optimizing glycemic control in patients with T1DM. The use of decision support systems provides a potential solution to allow for more frequent titration of insulin doses while minimizing the time required of providers and offers a promising addition to T1DM management. DreaMed Advisor is a decision support tool, approved by the FDA, that analyzes glucose values, insulin delivery, and food intake to provide clinical recommendations. Advice4U Pro is an ongoing randomized controlled trial seeking to show non-inferiority of the DreaMed Advisor system relative to experienced diabetes clinicians.

\section{6 | PLENARY SESSION-PREDICTION, PREVENTION, AND INTERDICTION OF T1DM}

To date, five immune therapies have been shown to modify T1DM progression (Abatacept, Anti-Thymacyte Globulin, Alefacept, Rituximab, and Tepalizumab) but no therapies are currently standard of care. A 2-week course of Teplizumab, an anti-CD3 antibody, demonstrated a median 2 -year delay in progression to T1DM $(P<0.05)$. A total of 25 of 44 participants were noted to be diabetes-free at completion of the study, with the longest follow up being a total of 88 months.

The Environmental Determinants of Diabetes in the Youth (TEDDY) study aims is to understand the genetic and environmental factors that influence the development of T1DM. Through the work of the TEDDY study, a genetic risk score involving a 41 SNP array has been developed and validated that combines HLA and non-HLA genes to identify children in the general population at risk for the development of multiple islet autoantibodies. This genetic risk score identifies $>10 \%$ of individuals by age 6 years and $>15 \%$ of individuals by age 10 years and while this might not be translatable to the general population, it could help identify a cohort of children at high risk for T1DM development that could be involved with future clinical trials aimed at strategies for T1DM prevention. In addition, TEDDY demonstrated that if children are identified as high risk for the development of
T1DM early, they are at a much higher likelihood of being diagnosed with T1DM in a safe manner with a lower HbA1c, higher baseline cpeptide, and fewer episodes of DKA.

\section{7 | NUTRITION SYMPOSIUM- CONTROVERSIES AND CONSIDERATIONS}

Despite the growing interest in very low carbohydrate (VLC) diets to aid in optimizing glycemic control in T1DM, there is little evidence to support their use to date and clinicians must be aware of the challenges of these diets. Depending upon the reference definitions, VLC diets can vary between $<10$ and $<26 \%$ of total daily energy intake. This is as opposed to consensus recommendations for 45 to $50 \%$ of daily energy intake coming from carbohydrates. At present, studies in adults and children have been limited by small numbers and design flaws. Several small studies in adults have demonstrated variable results about the benefit of VLC diets on glycemic control. Many do report a decline in HBA1c or improved TIR. However, of concern, both weight loss and possible cognitive decline were found in adults using a VLC diet. One case series in children found evidence of growth failure while following a VLC diet, likely due to a combination of inadequate caloric intake and reduced insulin administration. Another concern is that when diets begin to limit carbohydrate content, the energy intake from fat increases, which can result in prolonged post-meal hyperglycemia as well as abnormal lipid profiles. Some groups have reported an increase in hypoglycemic events. Chronic ketogenesis may impair hypoglycemia awareness, which can be dangerous in patients who may have diminished glucagon stores due to low carbohydrate intake. It is unknown if glucagon can still be a suitable rescue drug for these patients. The avoidance of healthy carbohydrates such as fruits, whole grains, and legumes increase the risk of nutritional deficiencies.

Ultimately, patients do not need VLC diets to achieve good glycemic control. Patients can attain glycemic targets by healthy eating routines with limited grazing, optimizing the insulin to carbohydrate ratio, administering insulin pre-prandially, choosing low glycemic index carbohydrates, and adding protein and fiber to meals.

\section{8 | PLENARY SESSION-DIABETES HISTORICAL PERSPECTIVES: YESTERDAY, TODAY, AND TOMORROW}

Our knowledge of diabetes mellitus continues to improve and change with research findings. DCCT irrefutably provided the link between hyperglycemia and microvascular disease. Other major progress in management of T1DM include the transition from urine glucose monitoring, to finger stick blood glucose monitoring, and now to CGM. Scandinavian countries have demonstrated both improved outcomes and improved access to diabetes technology-a goal for all countries to strive toward. 


\section{9 | SYMPOSIUM-CLOSING THE LOOP}

AID systems include a continuous glucose monitor input into a controller algorithm that can then adjust insulin delivery in order to maintain glucose values within a desired range while minimizing the need for user input. Although advances in AID and hybrid closed loop insulin delivery systems have improved, many challenges remain before a truly closed loop system can be developed including unannounced meals, physical activity, the on-body footprint, and logistics of the devices. Closed loop studies across the pediatric age range have highlighted the marked variability in daily insulin doses among pediatric patients, particularly in the youngest age groups. In older children, the need for user friendly systems was highlighted as current systems have resulted in high rates of attrition just 3 months after starting the Medtronic 670G. Studies exploring the impact of high intensity exercise have shown that these AID systems can improve control and minimize hypoglycemia in even the most demanding situations. While many AID systems are regulated and approved by government systems, there is a do-it-yourself community who engineer their own AID through protocols widely accessible on the internet. The two most widely known systems in use today are Loop and OpenAPS. While AID systems hold great promise, there is also concern that the costs of these technologies are widening the gap among patients of high and low socioeconomic status.

Finally, all presentations are available on the ISPAD website Conference Resources Platform: https://medialibrary.ispad.cyim.com/

How to cite this article: Addala A, March C, Marks B, et al. ISPAD Annual Conference 2019 Highlights. Pediatr Diabetes. 2020;21:152-157. https://doi.org/10.1111/pedi.12986 